\title{
The pen is mightier than the camera
}

\section{Medical drawings bring a new dimension to the Royal Academy's Summer Exhibition in London.}

\section{Martin Kemp}

Why would anyone involved in the sciences resort to drawing by hand? In this era of digital photography, which generates high-resolution images that can be manipulated at will, and of devices such as electron microscopes that can be set up to see and represent an object without fallible human intervention, why use such an apparently outmoded technique?

The answer is that the act of accurate drawing is still the best way to guarantee intense looking on the part of the artist, and, in terms of communication with the viewer, it provides a visual language of unprecedented focus, directness and dynamism. A drawing can transcend temporal limitations in a way that no other static image can. Data from different stages in a process or procedure can be overlaid yet remain clear, and anyone witnessing the making of the drawing can be taken step-bystep through what is being demonstrated.

Any artist who draws, or anyone who has sat for a drawn portrait, will not need telling that drawing involves the most intense visual concentration. It is a way of "learning how to see", to quote the first president of the Royal Academy, Joshua Reynolds. It is appropriate that when David Hockney and Allen Jones, former enfants terribles of the 1960s, were asked to organize a section devoted to drawing at this year's Royal Academy Summer Exhibition, they decided to include examples from outside Fine Art.

The drawings that have created the greatest stir — and won a prize — are by the heart surgeon Francis Wells of Papworth Hospital in Cambridge, UK. The example shown here illustrates the repair of a post-myocardial infarction ventricular septal

reveals much, much more: the buds are all surrounded by smaller buds, which in turn support even smaller ones, and so on. Homing in on the boundary of $M$ reveals a menagerie of multi-branched spirals, dragons and seahorses. Hairs of imperceptible fineness extend from the buds, holding along their lengths minute replicas of the entire Mandelbrot set.

Is the Mandelbrot set just a pretty curiosity? Far from it. It is a fundamental parameter information about nonlinear processes. First, the position of a complex number $c$ relative to $M$ tells us a great deal about the iteration of the quadratic mapping $f(z)=z^{2}+c$. The (filled-in) 'Julia set' at $c$ consists of those complex numbers $z$ whose iterates under repeated application of $f(z)=z^{2}+c$ never wander far from the origin. This Julia set comprises a single piece precisely when $c$ lies in the Mandelbrot set. (Interestingly, this topological dichotomy was noted by Pierre set that encodes an enormous amount of

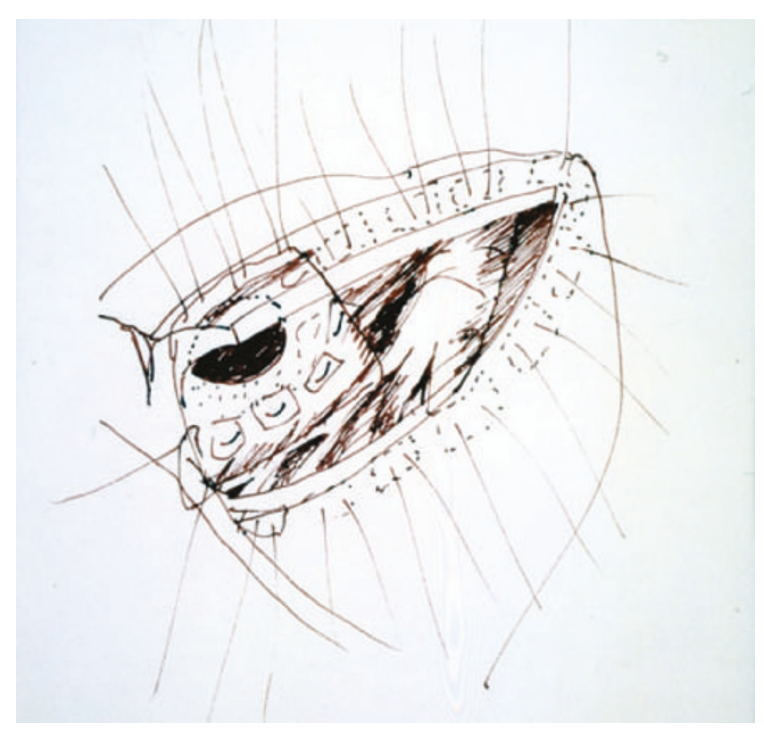

clutter that would be generated in a photograph or video. Even for someone who has not seen the drawing being made, the sense of the motion of the draughtsman's hand, in accordance with the flow of his ideas, is clear, just as it would be in a rapid sketch by Hockney or Jones. Indeed, Wells' sketch shares notable affinities with Hockney's drawings, especially those of the 1960s.

Wells himself is fully conscious of the special quality of the act of communicative drawing in time and place: "Having just completed the operation, drawing one's thoughts feels like an extension of the operation with the hand-eye interworking." This demonstrates defect. Wells explains that it illustrates "the incision in the left ventricle to get access to the ventricular cavities, the relationship of the papillary muscles and the underside of the atrioventricular valves, the lesions involved, in this case a hole in the septum, the placement of the sutures for the introduction of the patch to close the defect and the patch in place. Finally, the sutures necessary to close the incision are also shown."

The circumstances of its creation explain the impulsive character of the drawn line. This picture was drawn after the operation, over a cup of coffee in the surgeon's room, to explain the procedure to visiting and trainee surgeons. The speed and weight of line and the selective hatching are integral to the act of emphatic communication, picking out exactly what is essential, filtering out all the visual

Fatou and Gaston Julia in 1918-19, but it was many years before its real significance and delicacy was appreciated.)

Much more than this, the exact position of $c$ in $M$, such as the bud in which it lies, gives a very full description of how the iterates of $f(z)$ behave: for example, whether there are periodic cycles. Even more surprising is that, although defined in terms of the simplest of nonlinear maps, $f(z)=z^{2}+c$, the Mandelbrot set is 'universal' in that it underlies the behaviour of very large classes of more complicated nonlinear mappings, the likes of which crop up throughout modern mathematics and its applications.

The emergence of the Mandelbrot set in 1980 led to a flurry of activity among mathematicians trying to understand its structure and significance, resulting in some of the most impressive advances in pure mathematics in recent years. In 1982, Adrien Douady and John Hubbard proved the (far from trivial) fact that $M$ is connected, though "the superiority of drawing over other means of visual record, as it is a process of communicating thought and ideas as composed in the mind without the constraints of any equipment limitations."

It is interesting to witness moves to reintroduce drawing into the teaching of sciences and technologies. As Wells says, drawing is "powerful in teaching and can enhance explanation - acting out the move ... through the use of line. I feel this is very like the cognitive process in the artist, who will look and look before committing a line to paper."

The drawings are on show at the Summer Exhibition of the Royal Academy of Arts in London until 16 August.

Martin Kemp is professor of the history of art at the University of Oxford, Oxford OX1 1PT, UK, and co-director of Wallace Kemp Artakt. it is still unknown whether $M$ is locally connected - can you travel between nearby points of Mstaying inside $M$ without making too long a detour? In 1998, Mitsuhiro Shishikura showed that the boundary of $M$ has fractal dimension 2, which means that it is just about as complicated as can be, though it is still not known whether this boundary has positive area.

This is the fourth volume of Mandelbrot's Selecta, comprising edited reprints of the author's papers. Largely from the 1980s, these include the series of seminal papers that revealed the magnificence and omnipresence of the Mandelbrot set, together with other papers related to the iteration of functions. Several sections provide an overview of the work along with its scientific and historical background. One chapter has been written specifically to help the nonexpert appreciate the rest of the book.

Much of the material does not require particularly technical knowledge, so the 